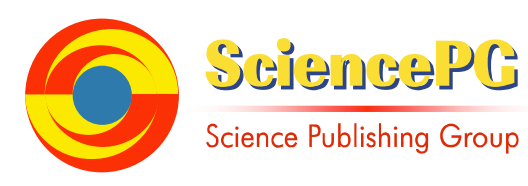

\title{
Performance Simulation and Fabrication of PZT Piezoelectric Composite Ring
}

\author{
Wang Hong-wei \\ Science College, Beijing Information Science and Technology University, Beijing, China
}

Email address:

drhwh@bistu.edu.cn

To cite this article:

Wang Hong-wei. Performance Simulation and Fabrication of PZT Piezoelectric Composite Ring. International Journal of Materials Science and Applications. Vol. 5, No. 2, 2016, pp. 89-94. doi: 10.11648/j.ijmsa.20160502.19

Received: February 29, 2016; Accepted: April 14, 2016; Published: May 4, 2016

\begin{abstract}
High-frequency wideband omnidirectional transducer is the development direction of the research of underwater acoustic transducer, PZT piezoelectric composite ring is not only capable of horizontal omnidirectional transmitting and receiving underwater acoustic signal. Meanwhile, it also makes the bandwidth of the transducer is expanded. Using the ANASYS finite element simulation software, the modal simulation of the ring structure of the 2-2 type piezoelectric composite is carried out, and the relationship curve of the bandwidth and the structure size is obtained. Based on the simulation, a design scheme is formed, cutting circular piezoelectric ceramics along the axial direction, there is epoxy resin in the groove. Thus the 2-2 type piezoelectric composite circular tube is formatted. The piezoelectric composite circular tubes suitable for horizontal omnidirectional broadband transducer.
\end{abstract}

Keywords: 2-2 Piezoelectric Composite, Simulation, Fabrication, Properties

\section{Introduction}

Composite materials are multiphase materials, which are made of piezoelectric phases (such as PZT) and polymer phases. It overcomes the shortcomings of the single-phase piezoelectric material and retains the strong piezoelectric property of piezoelectric phase material with a lower Qm value. The bandwidth of the transducer element is inversely proportional to the $\mathrm{Qm}$ value of the material, so the piezoelectric composite material is especially suitable for making the wide band transducer [1-6]. The typical piezoelectric composite material is made by cutting the ceramic and casting polymer. This method makes planar array composites that the piezoelectric ceramic is cut into the ceramic column array and the flexible polymers (such as epoxy resin, rubber, etc.) are added in the ceramic column array.

Chen Junbo et al. Of the No. 715 Research Institute of China Shipbuilding Heavy Industry Group made a piezoelectric composite piston transducer and the same size piezoelectric ceramic transducer [3]. The composite exhibits a single thickness resonance that the frequency is $180 \mathrm{kHz}$ and the $3 \mathrm{~dB}$ bandwidth is $15 \mathrm{kHz}$. However, the piezoelectric ceramic presents multi-peaks resonance and the maximum resonance bandwidth is only $5 \mathrm{kHz}$. The bandwidth of piezoelectric composite is 3 times that of single phase piezoelectric ceramic.

1-3-2 type piezoelectric composite broadband underwater acoustic transducer [7] was developed by Xian Xiaojun et al, who are in the institute of applied acoustics in Shanxi Normal University. The transducer is placed in anechoic test pool to test Water emission voltage response. The maximum response voltage of 1-3-2 type piezoelectric composite ceramic material underwater acoustic transducer is $183 \mathrm{~dB}$, and $-3 \mathrm{~dB}$ bandwidth of nearly $40 \mathrm{kHz}$. Its bandwidth is much larger than that of the ceramic.

British S. Cochran used the 1-3 single crystal piezoelectric composite and matching layers to produce a bandwidth of doubling frequency of the underwater acoustic transducer [8]. Turkey I. Ceren Elmash has made a wide bandwidth and wide beam acoustic transducer based on 1-3 piezoelectric composites. The transducer can be applied to the field of underwater acoustic communication [9]. South Korea's Tian Zhi Fabricated a wide band ring transducer which is based on piezoelectric composite material with a matched layer [10].

At present, most of the curved transducers use piezoelectric ceramics as the sensitive material, because its $\mathrm{Q}$ value is much larger than that of the composite material. that is, the bandwidth of the material is less than that of the 
composite material. Therefore, if the composite material is made into a curved surface, the bandwidth of the transducer is bound to expand and the large open-angle beam radiation Sonic can be realized.

\section{Performance Parameters of PZT-52 Piezoelectric Ceramic Ring with Different Thickness}

The structure of the piezoelectric ceramic ring is shown in Figure 1 . The Outer diameter is $2 r_{2}$, Inside diameter is $2 r_{1}$, The height is $\mathrm{h}$, The thickness is $\mathrm{t}=r_{2}-r_{1}$, The four kinds of piezoelectric ceramic ring has been tested which Outer diameter is $25 \mathrm{~mm}$, the height is $3 \mathrm{~mm}$, the inner diameter is $16 \mathrm{~mm}, 17 \mathrm{~mm}$,
$18 \mathrm{~mm}, 19 \mathrm{~mm}$, The instruments used in the test are Agilent 4294A (40Hz-110MHz), Test results are shown in Table 1.

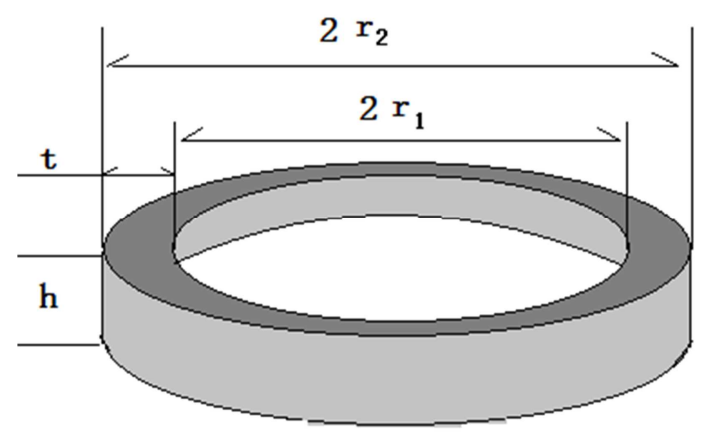

Fig. 1. The geometry of Piezoelectric ceramic ring.

Table 1. Performance parameters of PZT-52 ceramic ring with different thickness.

\begin{tabular}{|c|c|c|c|c|c|c|c|c|c|c|}
\hline \multirow{2}{*}{$\begin{array}{l}\text { Model: } \\
\varphi 25 \times \varphi 16 \times 3\end{array}$} & \multicolumn{10}{|c|}{ Number (PZT-52) } \\
\hline & $1 \#$ & $2 \#$ & 3\# & 4\# & $5 \#$ & $6 \#$ & 7\# & $8 \#$ & 9\# & $10 \#$ \\
\hline resonant frequency $f(\mathrm{kHz})$ & 313 & 314 & 313 & 313.8 & 314.5 & 314.5 & 313.8 & 314.5 & 315.8 & 313.8 \\
\hline Bandwidth (kHz) & 3.3 & 3.09 & 2.86 & 2.76 & 3.82 & 3.3 & 2.6 & 3.3 & 2.52 & 2.6 \\
\hline $\mathrm{Q}$ value & 94 & 101 & 110 & 113 & 81.7 & 94.8 & 120.9 & 93.7 & 126.5 & 118 \\
\hline peak value of conductance $\mathrm{G} / \mathrm{mS}$ & 41 & 43 & 45 & 44.5 & 37.8 & 41.23 & 45.2 & 41.54 & 42.3 & 45.8 \\
\hline Model: & \multicolumn{10}{|c|}{ Number (PZT-52) } \\
\hline$\varphi 25 \times \varphi 17 \times 3$ & $1 \#$ & $2 \#$ & $3 \#$ & $4 \#$ & $5 \#$ & $6 \#$ & $7 \#$ & $8 \#$ & $9 \#$ & $10 \#$ \\
\hline resonant frequency $f(\mathrm{kHz})$ & 341 & 340 & 343 & 342 & 343 & 345 & 343 & 342 & 342 & 342 \\
\hline Bandwidth (kHz) & 3.8 & 3.7 & 3.5 & 3 & 3.6 & 3.6 & 3.5 & 4 & 4 & 2.9 \\
\hline Q value & 89.7 & 89 & 96 & 114 & 93 & 95 & 98 & 83 & 84 & 115 \\
\hline peak value of conductance $\mathrm{G} / \mathrm{mS}$ & 49.5 & 50 & 54 & 55 & 50.7 & 48.5 & 52.9 & 46 & 46 & 55 \\
\hline Model: & \multicolumn{10}{|c|}{ Number (PZT-52) } \\
\hline$\varphi 25 \times \varphi 18 \times 3$ & $1 \#$ & $2 \#$ & $3 \#$ & $4 \#$ & $5 \#$ & $6 \#$ & $7 \#$ & $8 \#$ & $9 \#$ & $10 \#$ \\
\hline resonant frequency $f(\mathrm{kHz})$ & 373 & 373 & 372 & 374 & 372 & 375 & 374 & 374 & 374 & 374 \\
\hline Bandwidth (kHz) & 5.6 & 4 & 3.8 & 4.1 & 4.5 & 4.1 & 4.4 & 3.7 & 3.8 & 4.5 \\
\hline$Q$ value & 65 & 93 & 95 & 90 & 81 & 91.7 & 84 & 100 & 97 & 81.3 \\
\hline $\begin{array}{l}\text { peak value of conductance } \\
\mathrm{G} / \mathrm{mS}\end{array}$ & 51 & 62 & 64 & 61 & 59 & 57.7 & 55 & 59 & 62.5 & 57.7 \\
\hline Model: & \multicolumn{10}{|c|}{ Number (PZT-52) } \\
\hline$\varphi 25 \times \varphi 19 \times 3$ & $1 \#$ & $2 \#$ & $3 \#$ & 4\# & $5 \#$ & $6 \#$ & $7 \#$ & $8 \#$ & $9 \#$ & $10 \#$ \\
\hline resonant frequency $f(\mathrm{kHz})$ & 408 & 405 & 404 & 407 & 405 & 405 & 403 & 4.6 & 403 & 405 \\
\hline Bandwidth (kHz) & 5 & 4.2 & 4.75 & 5 & 4.8 & 4.9 & 4.3 & 5 & 5.9 & 4.8 \\
\hline$Q$ value & 81.6 & 95 & 85.9 & 80.9 & 83 & 81.5 & 93.6 & 80 & 68 & 83 \\
\hline peak value of conductance $\mathrm{G} / \mathrm{mS}$ & 68.4 & 78.9 & 71 & 65.2 & 69.3 & 68 & 77.7 & 67 & 64 & 69.5 \\
\hline
\end{tabular}

From the table1, we can see that the resonant frequency decreases with the increase of the thickness, the bandwidth decreases with the increase of the thickness, the $Q$ value increases with the increase of thickness, and the peak value of conductance decreases with the increase of thickness.

\section{PZT Piezoelectric Composite Ring Design and Simulation}

The geometry of the piezoelectric composite ring shows in Figure 2. Composite rings are made by cutting ceramic ring pouring epoxy - Sample polish - coated electrode process. A piezoelectric ceramic ring is cut by Accurate control of the knife gap and step to make composite ring arranged in a uniform array.

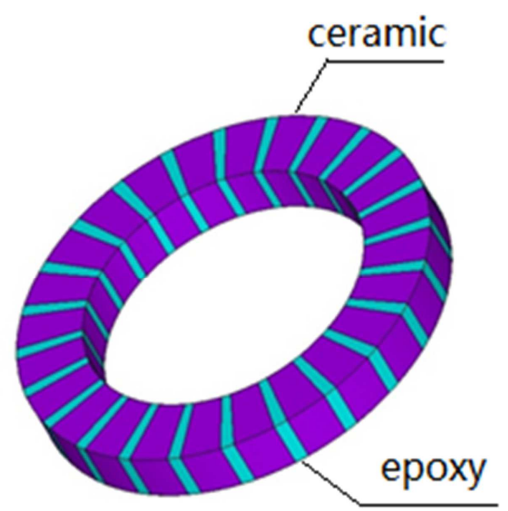

Fig. 2. The geometry of Piezoelectric composite ring. 
Ring structure parameters include the volume percentage of the composite piezoelectric ceramic $\mathrm{V}$, the radius of the circle $\mathrm{R}$, the thickness of the ring $\mathrm{T}$ and the height of $\mathrm{H}$. The volume percentage of the composite piezoelectric ceramic directly determines the electromechanical coupling coefficient $\mathrm{Kt}$ and Qm value of the composites.

In a cylindrical coordinate system modeling and simulation, it makes the piezoelectric ceramic that the height is $h$, the inner radius is $\mathrm{r} 1$, the outer radius is $\mathrm{r} 2$ and the polar angle is from $0^{\circ}$ to $8^{\circ}$. Then it makes the epoxy resin that the height is $\mathrm{h}$, the inner radius is $\mathrm{r} 1$, the outer radius is $\mathrm{r} 2$ and the polar angle is from $8^{\circ}$ to $10^{\circ}$. The entity is copied 36 parts by the method of rotational symmetry to make the entire annular composite ring. The thickness of the composite ring is $4 \mathrm{~mm}$,
The height of the composite ring is $3 \mathrm{~mm}$, the inner radius of the composite ring is $17 \mathrm{~mm}$. By simulation, the resonance frequency is $372 \mathrm{KHz}$. As shown in figure 3 .

By changing height $h$, thickness $t$, and the inner and outer ring radius, the ring resonant frequency and bandwidth are obtained with the variation in the size of its structure as shown in Figure 4.

From Figure 4, we can see that the ring resonant frequency decreases with the increase of the ring thickness $t$ and the height $\mathrm{h}$, and does not change with the change of the radius; The ring bandwidth $\mathrm{BW}$ also decreases with the increase of thickness $t$ and height $h$, and does not change with the change of radius.

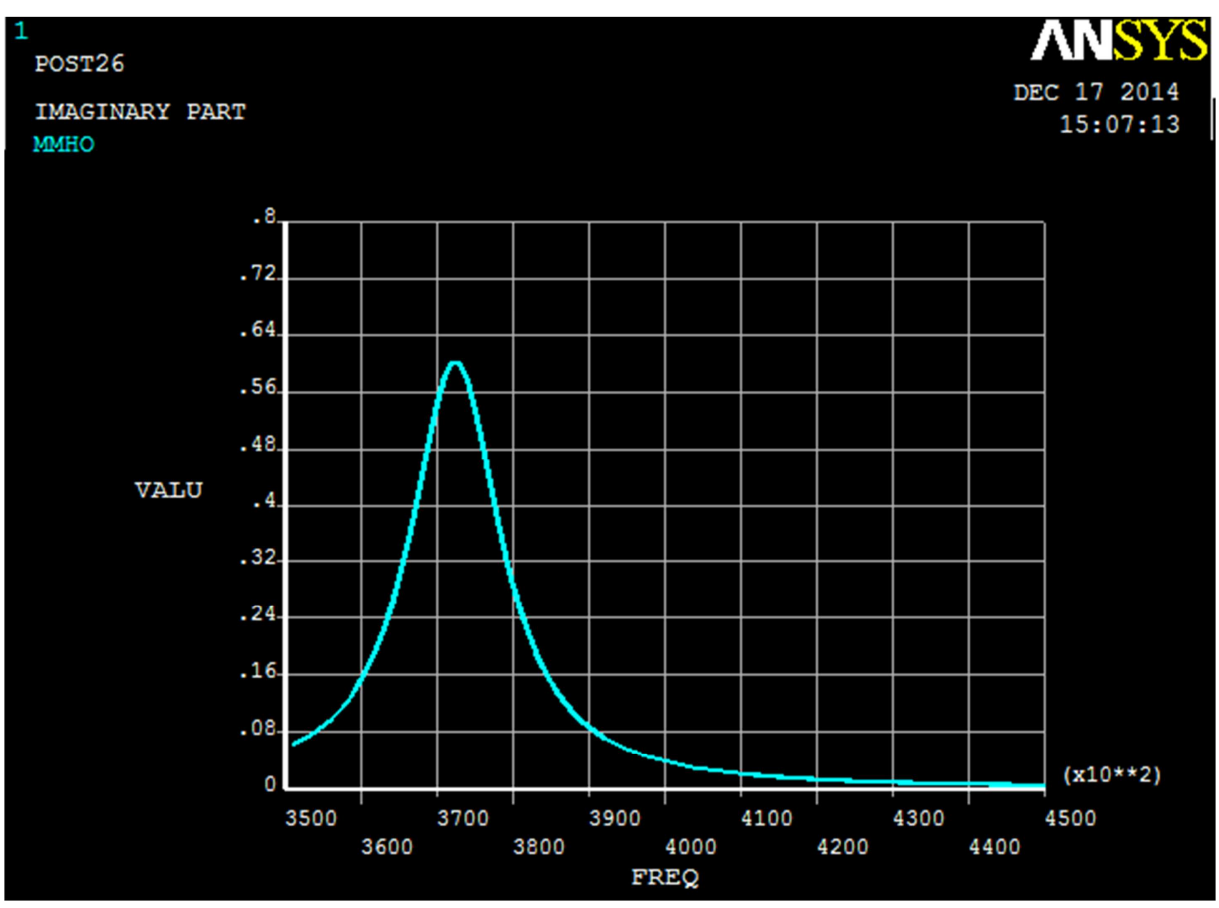

Fig. 3. The harmonic response analysis of the ring.

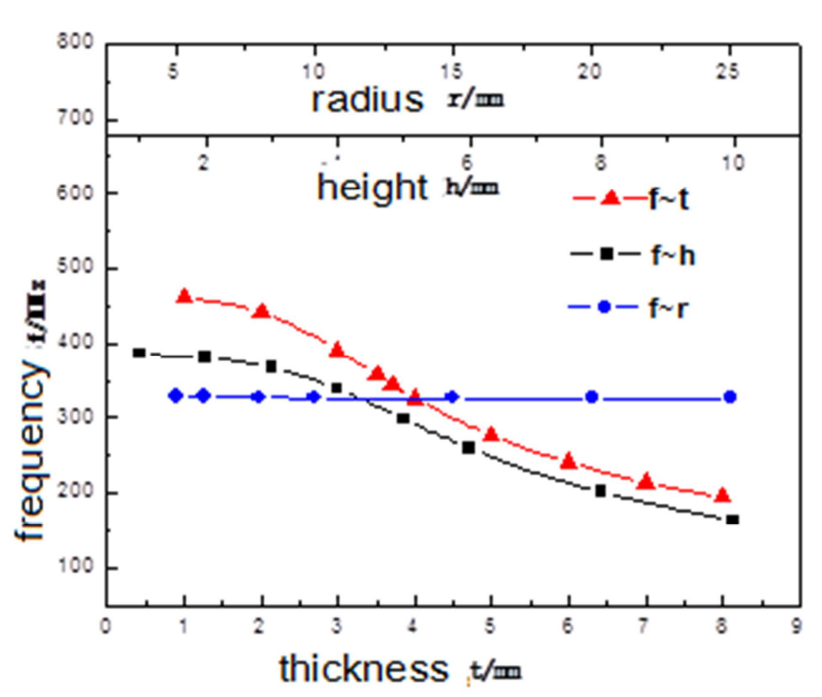

a) The law of the resonant frequency $f$ with the parameters of the ring

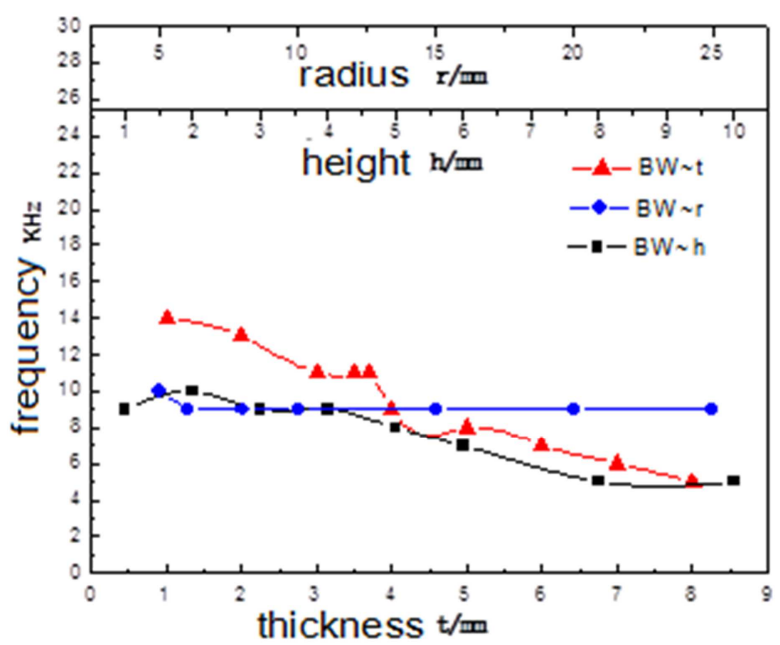

b) Bandwidth BW with different parameters of the ring

Fig. 4. The resonance frequency and bandwidth of the composite ring are changed with the parameters of the ring. 


\section{Preparation of PZT Piezoelectric Composite Ring}

On the basis of the conventional cutting - casting process, Composite rings are directly made by cutting ceramic ring - pouring epoxy - Sample polish - coated electrode process. Man continues to explore the preparation process. Especially a piezoelectric ceramic ring is cut by Accurate control of the knife gap and step to make composite ring arranged in a uniform array. the preparation and technical route of the composite ring is shown in figure5.

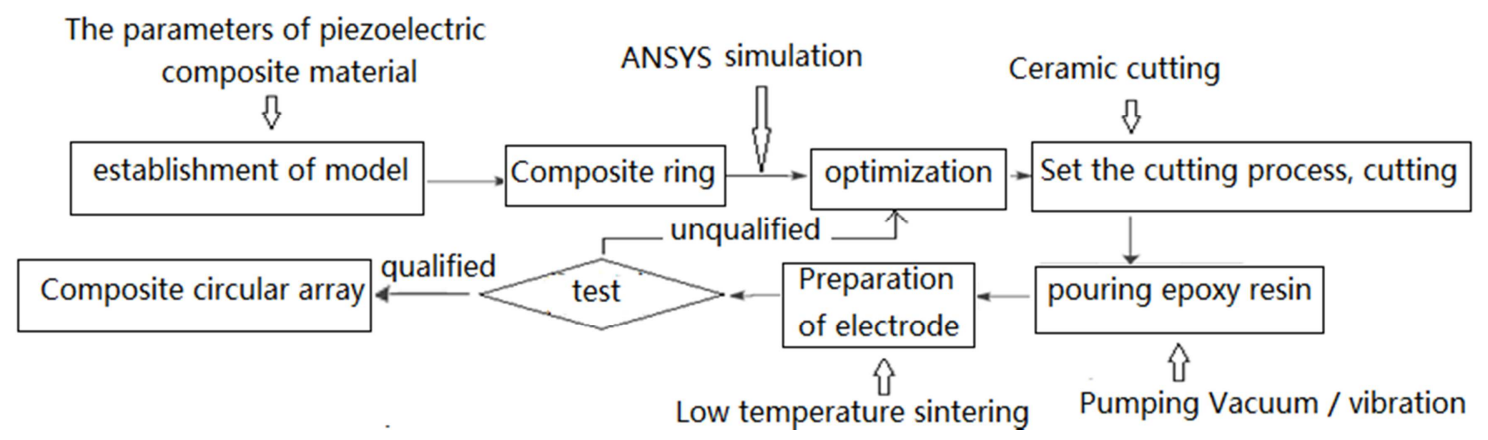

Fig. 5. The fabrication of composite ring array.

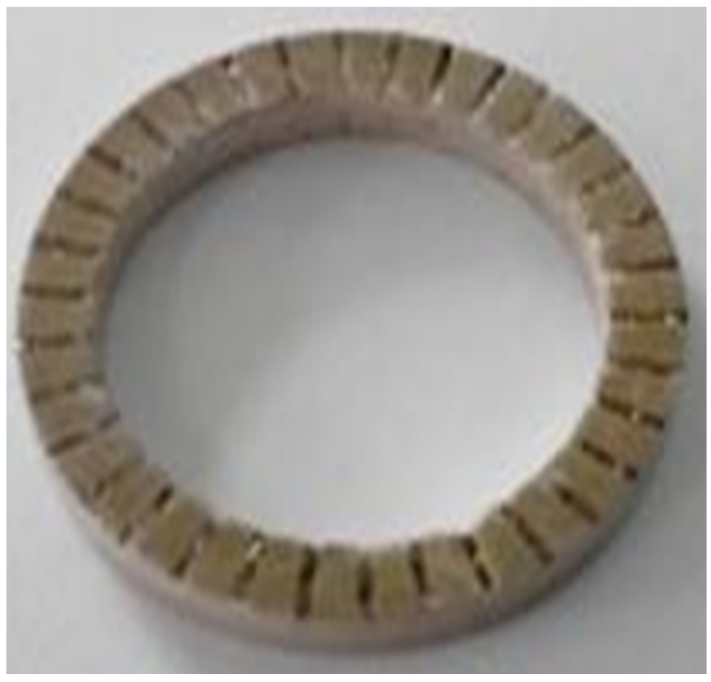

Fig. 6. Preparation of composite ring array.

The piezoelectric ceramic ring is PZT-5 production produced by the acoustics Institute of Chinese Sciences Academy. The bottom surface of the piezoelectric ceramic ring is placed on $1 \mathrm{~cm}$ thick square plates and is fixed with melted paraffin. After the paraffin solidification, the steel plate together with the ceramic ring are placed into the cutting positions in the disco automatic grinding wheel dicing machine of the Japanese company (model DAD321). Then setting programs in the cutting machine and cutting the ring into 36 parts. Epoxy resins are produced fluid, and the epoxy resins are poured in the ceramic skeleton. At the same time, the bubble is evacuated and the epoxy resin is evenly filled. After Curing at room temperature, the composite material is obtained. Finally Polishing rough shaping makes a composite material ring, and the inner and outer surface is plated electrode to make a piezoelectric composite ring.

Due to the preparation process of the polarization of the piezoelectric ceramic under normal temperature and atmospheric pressure, the effect of the electrode of the piezoelectric ceramic is little. So the polarization of the composite material is omitted. The composite material is made into a kind of ring as shown in figure 6 .

\section{Performance Test of the Piezoelectric Composite Ring}

The dielectric and piezoelectric properties of the composite ring are tested. When the test frequency is $100 \mathrm{~Hz}$, the piezoelectric constant $\mathrm{d} 33$ of the piezoelectric composites is measured by the piezoelectric constant tester (model: ZJ-3D) ;Vibration displacement of composite materials (Polytec Scanning Vibrometer) was tested by laser scanning vibration instrument; The low frequency capacitance and frequency characteristics of the composite material are tested by a precision impedance analyzer (4294A Agilent).

The sample parameters were measured, such as Piezoelectric constant, the vibration displacement (5V voltage drive), the relative dielectric constant, the speed of sound, acoustic impedance and so on. As is shown in table 2.

Table 2. Properties of 2-2 composite.

\begin{tabular}{|c|c|c|c|c|c|c|c|c|c|c|}
\hline Material & $d_{33}(\mathrm{pC} / \mathrm{N})$ & Shift (pm) & $\varepsilon_{\mathbf{r}}$ & Dielectric loss & $f_{\mathrm{s}}(\mathrm{kHz})$ & $f_{\mathrm{p}}(\mathrm{kHz})$ & Bandwith (kHz) & $v(\mathrm{~m} / \mathrm{s})$ & $\rho\left(\mathrm{kg} / \mathrm{m}^{3}\right)$ & Z (Mraly) \\
\hline $\begin{array}{l}\text { 2-2 type } \\
\text { piezoelectric } \\
\text { composites }\end{array}$ & 480 & 89.5 & 859 & 0.04 & 388 & 489 & 11.2 & 3200 & 5018.7 & 18.05 \\
\hline
\end{tabular}

From table 1, we can see that the piezoelectric ceramic ring bandwidth is $3.6 \mathrm{KHz}$, from table 2 , we can see that the 
piezoelectric composite ring bandwidth is $11.2 \mathrm{KHz}$ with the same dimensions of the ring. It is showed that the bandwidth can be increased by adding epoxy resin to the piezoelectric ceramic as a 2-2 type piezoelectric composite material.

The impedance characteristic of the piezoelectric composite ring is shown in figure 7 . The main vibration resonance of the composite material is much larger than other vibration, and the bandwidth is larger than that of the ceramic. These characteristics can improve the bandwidth and frequency selectivity of the transducer, so the design of the transducer array is easy to implement.

The test and simulation results are compared. The test resonant frequency $\mathrm{f}$ is $388 \mathrm{KHz}$ and the bandwidth $\mathrm{Bw}$ is $11.2 \mathrm{kHz}$. The simulation resonance frequency $\mathrm{f}$ is $372 \mathrm{kHz}$ and the bandwidth $\mathrm{Bw}$ is $9 \mathrm{kHz}$. The highest frequency difference is $4.3 \%$, and the bandwidth difference is $24.4 \%$.

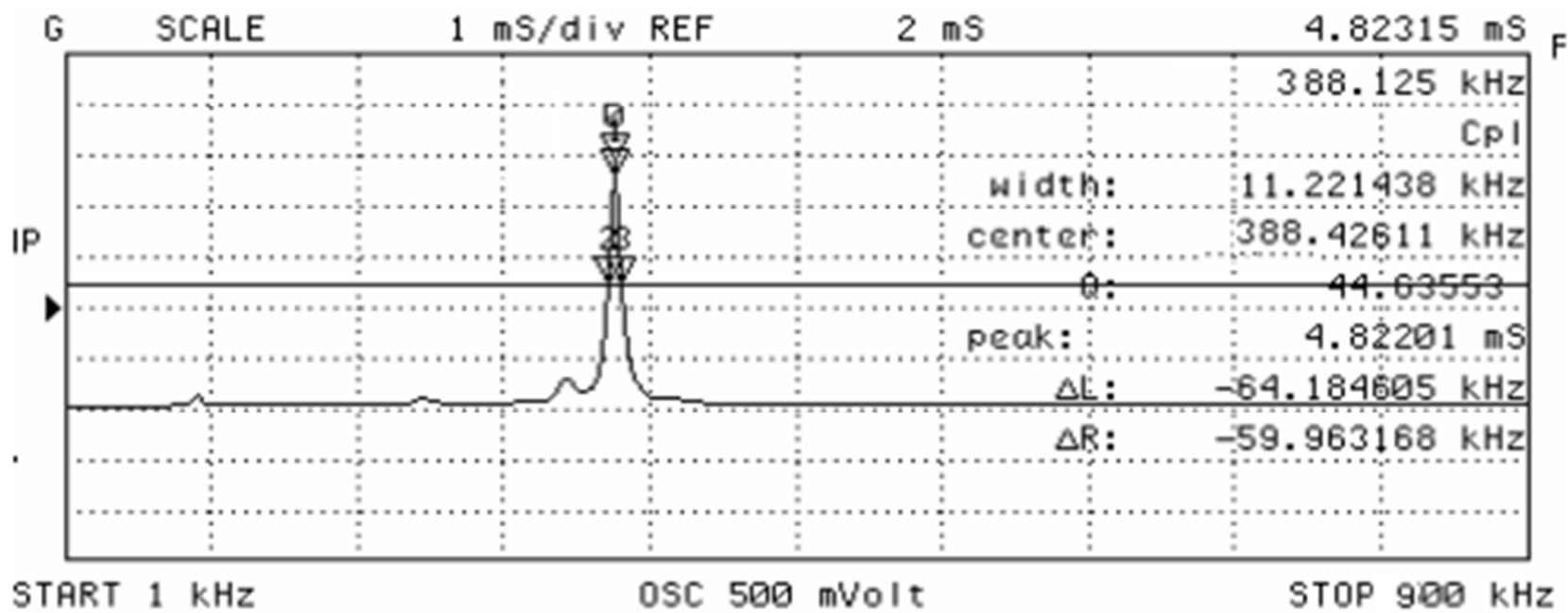

Fig. 7. Impedance characteristic curve of the ring of 2-2 type piezoelectric composite material.

The reason of the difference between the actual and the simulation bandwidth:

(1) The piezoelectric composite ring in the actual test has the internal energy loss of the material (his is due to the full compatibility of the ceramic and epoxy phases in the process, and the air is not fully discharged). Thereby that is more than the results of the simulation to expand the bandwidth.

(2) When the piezoelectric composite material is measured in the air, the air damping causes the energy loss of the transducer and also expands the bandwidth.

Above two reasons, the first is the main factor. At present, the bandwidth difference is up to $24.4 \%$. This shows that we should pay attention to fully mixing and exhaust in the epoxy pouring process.

\section{Conclusion}

The preparation technology of plane composite material is mature. Coated electrode can be fabricated by the flat screen printing technology, but curved surface electrode is difficult to be printed by the flat screen printing technology. So it is difficult to realize the Curved surface composite material and limits the beam angle of the transducer. In this paper, Composite ring array is directly made by the process of cutting ceramic ring - pouring epoxy - sample grinding curved screen coated electrode. By test, the resonant frequency is $388 \mathrm{KHz}$, the bandwidth is $11.2 \mathrm{kHz}$, the material piezoelectric constant $\mathrm{d} 33$ is $480 \mathrm{pC} / \mathrm{N}$, the vibration displacement is $89.5 \mathrm{pm}$, the sound speed is $3200 \mathrm{~m} / \mathrm{s}$, the Acoustic impedance is $18.05 \mathrm{Mraly}$, and the relative dielectric constant is 859 . The piezoelectric constant $\mathrm{d} 33$ and the vibration displacement of the 2-2 type composite can reach the performance of the piezoelectric ceramics, and the sound speed and the acoustic impedance are greatly reduced, the bandwidth is increased. If you want to further broaden the bandwidth, it can be two different frequencies of single-tube composite rings stacked to produce a coupling that can further broaden the Bandwidth.

\section{References}

[1] Li Li. 1 -3 type of piezoelectric composite materials and underwater acoustic transducer research. Beijing University of Posts and Telecommunications doctoral dissertation. May 2008.

[2] Bin Zhang, Likun Wang, Lei Qin etc. Influence of Volume Fraction of Piezoelectric Ceramics on Popertiesof PZT672/Epoxy1-3 Piezocomposite. Advanced Materials Research Vols. 989-994 (2014) pp 364-368.

[3] Chen Junbo, Wang Yuebing, Zhong Lin Jian. 1-3 type piezoelectric composite and ordinary PZT transducer performance contrast analysis. Acoustic and electronic engineering, 2007 (3): p 25-27.

[4] Sherman C H, Butler J L. Transducers and arrays for underwater sound [M]. Springer, 2007.

[5] Christopher J, Purcell A, Richard F. Multi-mode pipe projector: United States, 6584039 [P]. 2003-06-24.

[6] Alexander L. Butler, John L. Bultler-Multi Ply Resonant Wideband Transducer APP aratus, 2005, US6950373B2. 
[7] Xian Xiaojun, Lin Shuyu, Deng pan Wang, Ma chin Yi. Based on 1-3-2 type piezoelectric composite electric broadband acoustic transducer, piezoelectric and acoustooptic, Vol 36 No. 4, August 2014. P 491-497.

[8] Coehran S, Parker M, Franeh P M. Ultrabroadband single crystal composite Transducers for underwater ultrasound. IEEE Ultrasonics Symposium. Netherlands, 2005: 231-234P.
[9] Elmash I C, Koymen H A. A wideband and a wid-beamwidth acoustic transducer design for underwater acoustic communications. OCEANS 2006-Asia Pacific. SingaPore, 2007: 1-5P.

[10] Zhi T, Yongae R, Wonho K. Optimal design of an underwater Piezocomposite ring transducer. IEEE Ultrasonic symposium. China, 2008: 1405-1408P. 